\title{
MICHAE KRZYKAWSKI
}

Université de Silésie

\section{Derrida à l'œuvre. Autour de Circonfession et au-delà ${ }^{1}$}

our circonscrire chez Derrida cet « espace du flou Cahiers ERTA nous a proposé comme point de départ pour la réflexion, il faut voir l'auteur de Circonfession tel qu'il est à I'œuvre, et du coup insister sur la valeur autobiographique de ses écrits. Le sens du texte derridien est à chercher dans le so n de son style qui fait parler, malgré tout, ce que Derrida éprouvait comme malaise : l'impossibilité de se dire et d'en faire un récit ${ }^{2}$. Non pas qu'il soit dépourvu de mémoire, mais comme s'il en avait trop. Garder la mémoire des événements est une chose, savoir en parler en est une autre. Se prenant pour "l'amoureux de Mnemosyne ", Derrida pose une série de questions :

Mais que se passe-t-il quand l'amoureux de Mnemosyne n'a pas reçu le don du récit ? Quand il ne sait pas raconter une histoire ? Quand c'est précisément parce qu'il garde la mémoire qu'il perd le récit ?3

\footnotetext{
${ }^{1}$ Cet article s'inscrit dans le cadre du projet « Au nom de l'amitié, pour le bien de la communauté. L'amitié et la communauté dans la pensée contemporaine française ", réalisé grâce à l'aide financière du Centre National de la Science (NCN), Pologne. Projet n 2015/17/D/HS2/00512 (Sonata).

2 « Je n'ai jamais su raconter une histoire. Et comme je n'aime rien tant que la mémoire, et Mémoire elle-même, Mnemosyne, j'ai toujours ressenti cette impuissance comme une triste infirmité. Pourquoi suis-je privé de la narration ? Pourquoi n'ai-je pas reçu ce don de Mnemosyne ? " (J. Derrida, Mémoires pour Paul de Man, Paris, Galilée, 1988, p. 27).

3 Ibidem.
} 
Dans ce qui suit il s'agira d'apporter une réponse possible. Je répondrai donc à ces questions en trois étapes. Tout d'abord, je montrerai que la sur-mémoire derridienne, qui rend le récit impossible, se manifeste également à travers une approche spécifique à la langue française. Derrida désire se $s$ a u ve $r$ en se disséminant à l'intérieur même de sa pensée. II rejette son c o r p s pour devenir son c o r p u s, son écriture disséminatrice lui étant la seule possibilité d'écrire son autobiographie impossible. En effet, ne pas savoir raconter une histoire n'implique pas de renoncer à l'autobiographique, loin s'en faut. II $s^{\prime}$ agit désormais de mettre cette impossibilité en œuvre et à l'œuvre ; monter un spectacle de soi, se performer sur la scène philosophique. Après tout, émouvoir. Le cas de Derrida, et ce sera la deuxième étape de ma réponse, me servira de tremplin pour m'interroger sur le rapport entre l'autobiographique et le philosophique dans le cadre d'un projet de recherche plus vaste qui porte sur la " philosophie auto/biographique ". Dans ce projet, Derrida occupe une position centrale. D'un côté, il est un fournisseur de méthode qui oriente la lecture et encourage à poser des questions à l'égard des textes écrits par d'autres philosophes. De l'autre côté, il est un cas à traiter en tant que celui qui, dans son écriture, a mis en question la frontière entre le plus privé et le public. En dernière étape, j'entamerai une lecture de Circonfession à partir de ce que j'appelle "fil pénien ", montrant quelques stratégies pour sauver dans sa propre pensée rapportée par un autre des traces de soi. 


\section{"Électroencéphalocardiosomatopsychogramme général »}

Dans une émission sur France Culture en 1998, Derrida avouait :

Au fond, mon désir d'écrire est celui d'une chronique exhaustive. Qu'estce qui me passe par la tête ? Comment écrire assez vite pour que tout ce qui me passe par la tête soit gardé ?4

Qu'une telle chronique n'ait jamais vu le jour, Derrida l'éprouvait comme "le regret de [s]a vie $\|^{5}$ tout en avouant, dans le même entretien, que son écriture a toujours eu une forte composante autobiographique. En fait, la distinction qu'on fait souvent entre ses premiers textes théoriques, qui sont la base de ce qu'on aura reconnu comme déconstruction ${ }^{6}$, et ses textes tardifs, que l'on définit, faute de mieux, comme littéraires, est fausse à bien des égards, car les uns et les autres sont originaires du même " désir d'écrire ". Ce qui change, ce

n'est pas le rapport à l'autobiographie ou le passage des textes non autobiographiques à des textes autobiographiques, mais une certaine modulation, une certaine transformation du ton et du régime de l'autobiographie. ${ }^{7}$

II devient donc possible, et c'est Derrida lui-même qui semble encourager une telle relecture, de lire ses philosophèmes comme des formes d'autobiographie et de suivre

4 J. Derrida, Sur parole. Instantanés philosophiques, Paris, Éditions de l'Aube, 1999, p. 18.

${ }^{5}$ Ibidem.

${ }^{6}$ Le terme de déconstruction est, bien sûr, celui de Derrida. Or, il est loin de jouer le rôle d'un concept central ou organisateur de sa pensée dans ses premiers écrits. Si la déconstruction est devenue une marque que l'on associe à la philosophie derridienne, cela est dû à la réception de sa pensée aux États-Unis à partir des années 1970. II est possible de parler de l'effet-Derrida qui faisait de la déconstruction une méthode de lecture que l'on croyait pouvoir appliquer à toutes sortes de studies qui se réclamaient de Derrida en tant que caution théorique.

7 J. Derrida, Sur parole, op. cit., p. 10. 
Derrida après ses traces dans lesquelles on peut voir des " lieux de mémoire ».

Ainsi faut-il placer l'autobiographie derridienne sous le signe de la diffé $r$ a n ce dans ses rapports étroits à la mémoire. Derrida ne cesse de s'inventer à travers son écriture, cette invention n'étant pourtant qu'une remémoration impossible. Dans l'un de ses premiers textes, Derrida fait une lecture des premiers travaux de Freud dans lesquels le futur père de la psychanalyse tente d'expliquer le travail de la mémoire selon un modèle neurologique du fonctionnement de l'appareil psychique, en introduisant le terme de frayage [Bahnung]. Une trace mnésique se fraie un chemin par effraction mais, ce chemin faisant, elle doit vaincre une certaine résistance. Or I'hypothèse freudienne intéresse Derrida " comme un modèle métaphorique et non comme une description neurologique ${ }^{8}$. En intervenant dans la pensée freudienne, Derrida remarque que " la mémoire n'est donc pas une propriété du psychisme parmi d'autres, elle est l'essence même du psychisme. Résistance et par là même ouverture à l'effraction de la trace ${ }^{9}$. Le frayage est réinterprété ici par le biais de la d i f fér a n c e. S'il y a une effraction, il faut qu'il y ait eu une résistance. Derrida semble dépister dans la métaphore freudienne sa propre métaphore du " faisceau » qui devait décrire son concept de base ${ }^{10}$. Des différences originaires produiraient alors leurs effets qui ne peuvent être intégrés par la conscience car elles sont antérieures à l'origine même de la mémoire. II s'agit désormais pour Derrida de penser (et de se remémorer) à partir de ce qui se situe hors mémoire, de faire recours à " une non-mémoire absolue ${ }^{11}$ qu'il aura dépeinte, de

8 J. Derrida, "Freud et la scène d'écriture », [dans :] Idem, L'écriture et la différence, Paris, Seuil, 1967, p. 298.

${ }^{9}$ Ibidem, p. 299.

10 J. Derrida, "La différance ", [dans :] Idem, Marges de la philosophie, Paris, Minuit, 1972, p. 3-4.

11 J. Derrida, "II n'y a pas le narcissisme (autobiophotographies) ", [dans :] Idem, Points de suspension, Paris, Galilée, 1992, p. 222. 
manière plus poétique, avec la métaphore de la cendre ${ }^{12}$. Cette non-mémoire, qui revient à la s u $\mathrm{r}-\mathrm{m}$ é $\mathrm{m}$ o i $\mathrm{re}$, car elle s'ouvre à tout ce qui s'oublie, est une origine pulsionnelle de l'écriture derridienne. Dans un autre entretien de 1983, c'est-à-dire de l'époque où la question de la mémoire, ainsi que celle du deuil et de la spectralité, devient littéralement sa hantise, Derrida dit :

mon désir premier n'est pas de faire œuvre philosophique ou œuvre d'art, c'est de garder la mémoire. Imaginons cette machine, par définition impossible, qui serait comme une machine à engrammer tout ce qui se passe, de sorte que les moindres pensées, les moindres mouvements du corps, les moindres traces de désir, le rayon de soleil, la rencontre de quelqu'un, une phrase en passant, soient inscrits quelque part, qu'un électroencéphalocardiosomatopsychogramme général soit possible, à ce moment-là mon désir serait absolument exaucé. ${ }^{13}$

Une telle mémoire, la sur-mé moire, défie toute tentative d'appropriation. Ma propre mémoire n'est jamais la mienne. D'un côté parce que ce qui reste à dire est hors mémoire et de l'autre côté parce que ce qui reste à dire ne peut se dire qu'avec un sentiment de perte. En effet, ce qui reste à dire ne m'appartient pas dès que cela est dit, qu'il n'y a plus de résistance mais une voie frayée. Dans Mémoires pour Paul de Man Derrida rappelle l'opposition entre un souvenir (Errinerung) et la mémoire pensante (Gedächtnis) que ce premier fait en travaillant sur l'Encyclopédie de Hegel. Rappelant de Man commentant Hegel, Derrida écrit :

Gedächtnis, c'est à la fois la mémoire qui pense [...] et la mémoire volontaire, la faculté mécanique de mémorisation; tandis que Erinnerung, c'est la mémoire intériorisante, le souvenir " comme recueillement intérieur et comme conservation de l'expérience $»{ }^{14}$

\footnotetext{
12 J. Derrida, Feu la cendre, Paris, Des Femmes, 1986. Cf. M. Krzykawski, "La mélancolie de la déconstruction. Ce qui reste de Jacques Derrida ", [dans :] Alkemie, 2017, n 19, à paraître.

13 J. Derrida, "Dialangues », [dans :] Idem, Points de suspension, op. cit., p. 153.

14 J. Derrida, Mémoires pour Paul de Man, op. cit., p. 64.
} 
La mémoire pensante est dirigée vers l'extérieur où demeure ce qui ne se symbolise pas et, par conséquent, ne peut pas devenir un souvenir, le sen $\mathrm{s}$ d'une expérience intériorisée. Son pouvoir est « p r é o c c u p é par un passé qui n'a jamais été présent et ne se laissera jamais ranimer dans l'intériorité d'une conscience ${ }{ }^{15}$. Pour Derrida, écrire revient à garder la mémoire dans cette double injonction entre l'effraction et la résistance, entre le souvenir et la mémoire pensante. Si tout son effort consiste à trouver un idiome qui puisse témoigner de ce passé qui n'a jamais vu le jour, c'est aussi dans ce passé immémorisable qu'il ne cesse de se remémorer. Son idiome lui est le seul lieu vivable dans lequel vivre revient à sur-vivre.

\section{Le s o n du style, le s e n s du texte}

Quand on aborde l'écriture derridienne à partir de la manière dont il abordait la mémoire, on voit bien à quel point cette écriture, depuis ses premiers textes, est une recherche de soi, menée par celui qui se définit, par exemple, comme " une sorte de métis européen sur-acculturé, sur-colonisé ${ }^{16}$. Derrida est à retrouver dans son écriture dans ce va-et-vient entre les concepts qui deviennent pour lui le lieu d'identification et son expérience autobiographique qui inspire les concepts. II ne peut vivre qu'en se réécrivant et s'inventant dans une répétition perpétuelle. Manier la langue est pour lui une stratégie qui lui permet de sur-vivre dans son idiome singulier et à la mesure même de sa propre pensée qui est bien de langue française.

Afin de suivre l'inscription de Derrida lui-même dans sa propre pensée où la catégorie du p ro p re est mise en question ${ }^{17}$, il faut le prendre pour un auteur qui ne cesse

\footnotetext{
15 Ibidem, p. 77.

16 J. Derrida, L'autre cap, Paris, Minuit, 1991, p. 13.

${ }^{17} C^{\prime}$ est à travers son analyse de la poésie de Ponge que, " ne séparant plus, dans le propre, les deux tiges de la propreté et de la propriété » il
} 
d'écrire ni de chercher à se retrouver dans s a langue. II faut enfin I e dire en français derridien, apprendre à rendre justice au s o n de son style, j'y reviendrai, que l'on a trop souvent qualifié d'illisible. "J'ai pour cette langue [...] un amour inquiet, jaloux et tourmenté ", dit-il dans son long entretien avec Élisabeth Roudinesco, en insistant sur une attention constante à un certain mouvement de la phrase, à un travail, non pas du signifiant, mais de la lettre, de la rhétorique, de la composition, de l'adresse, de la destination, de la mise en scène, qu'il aurait partagée avec Lacan, même si celui-ci est "si français, mon Dieu, tellement plus que moi ! ». Dire Derrida, I e dire en parlant français, en faisant parler s o $\mathrm{n}$ français reviendrait à suivre (après) " une façon de t o u c h e r à la langue française, ou de se laisser toucher par elle ". Dire Derrida reviendrait donc aussi à reconnaître en quoi il est différent, dans sa manière de manier la langue, de Foucault, Lévi-Strauss, Deleuze, Althusser ou Lyotard qui " entretiennent avec la langue française - malgré les différences de style - une relation commune. Et au fond très paisible, très sédentaire. Tous, ils écrivent un "certain français" [...] : "ils" sont tous plus "français" que moi, et plus français les uns que les autres, et pourtant j'oserai prétendre qu'entre la langue "française" et moi, il y aura, il y aura eu plus d'amour. D'amour fou, si vous voulez. Et de jalousie, de jalousie réciproque, si cela ne paraît pas trop insensé ! ${ }^{18}$.

Chez Derrida, tout se joue autour du style de son écriture. Or, lorsqu'on écoute ses conférences ou qu'on le lit à haute voix, on remarque que son écriture est surtout à e ntendre. Mais cela n'est en rien surprenant. Si

évoque ce " quelque chose qui, dans le propre, dans la structure même du propre, ne se produit qu'à passer dans son autre, à se mettre en abyme, à s'inverser, à se contaminer, à se diviser. Et que là est la grande affaire de la signature » (J. Derrida, Signéponge, Paris, Seuil, 1988, p. 28-30).

18 J. Derrida, É. Roudinesco, De quoi demain... Dialogue, Paris, Flammarion, 2001, p. 30-31. 
Derrida a inauguré sa pensée en dénonçant le caractère phallogocentrique de la métaphysique occidentale qui privilégie la parole et ne fait de l'écriture que son piètre reflet, ce n'était pas pour inverser l'ordre de l'opposition et pour donner la primauté à l'écriture. II s'agissait bien de montrer que la parole doit faire recours à l'écriture pour être comprise ${ }^{19}$. On aura compris que l'écriture, quant à elle, passe le mieux par la parole qui lui assure sa dramaturgie. "La scène de l'écriture " reconnue chez Freud finit par devenir une écriture scénique.

Le son de son style, donc : il faut l'entendre, mais aussi il faut le bien manger ${ }^{20}$ pour mieux voir ce qui alimente sa pensée. (Et faut-il rappeler que le son, lui, se mange et qu'il est sain, même si l'homme, viril et carnivore, s'en sert pour nourrir les animaux dont il se distingue comme de sa partie intime qu'il ne veut pas reconnaître ? Faut-il rappeler que l'homme ne mange pas bien ? Faut-il rappeler, enfin, que Derrida, se recherchant et se perdant dans son écriture, se suit après les traces de son " animal autobiographique $»^{21}$ ?). Qu'il me soit permis de le suivre au plus près possible pour bien manger sa parole, car "le texte lu ne suffit pas, faut le manger, le sucer, comme le prépuce ${ }^{22}$. Reste pourtant à savoir s'il est possible de le suivre sans l'imiter: ne pas I' i m it e r pour ne pas li m ite r l'autonomie d'un lecteur de Derrida que je suis. Richard Rorty a bien raison de remarquer que l'objectif de Derrida est de devenir autonome par son écriture, en quoi il n'est en rien différent d'écrivains tels que Yeats ou Proust. Et ce serait

${ }_{19}$ Cf. J. Derrida, "La pharmacie de Platon ", [dans :] Idem, La Dissémination, Paris, Seuil, 1972.

20 J. Derrida, " "Il faut bien manger" ou le calcul du sujet ", entretien avec Jean-Luc Nancy, [dans :] Idem, Points de suspension, op. cit., p. 269-301.

21 J. Derrida, L'animal que donc je suis, Paris, Galilée, 2006, p. 17.

22 J. Derrida, Circonfession, [dans :] G. Bennington, Jacques Derrida, Paris, Seuil, 1991, p. 77. 
à nous, lecteurs de Derrida, de " nous détenir et profiter de cette écriture - voir quel confort ou exemple elle peut nous offrir et si elle est en rapport avec nos propres tentatives de devenir autonomes ${ }^{223}$. Or, pour devenir un lecteur autonome en lisant Derrida, il faut peut-être faire autrement et le lire de la manière la plus at te n tionn é e, c'est-à-dire doubler l'enjeu qui est le sien : lui être fidèle et infidèle à la fois ${ }^{24}$. Comme si (comment utiliser cette tournure sans saluer Derrida et sans y sentir sa présence spectrale ${ }^{25}$ ? Je l'imite à peine ma phrase entamée) l'autonomie passait par la fidélité. Je commence, donc, par l'imitation pour mettre ce principe à l'œuvre. L'autonomie, la sienne, la mienne et celle d'un tout autre lecteur, d'une tout autre lectrice, n'est pas à atteindre. Elle est plutôt à entendre. Nous devons nous entendre pour devenir a utonomes. Comme si, pour mieux voir ce que Derrida a d'inimitable, il fallait faire plus que voir ce qui est à voir dans son écriture ; comme s'il restait à y entendre le son de son style qui détermine le sens de son texte. D'un côté, je fais donc recours à l'écriture en courant après Derrida tel que je le se ns. Au fond, seule l'écriture permet de sentir le sens et de le distinguer, ce Derrida élidé ${ }^{26}$ qui nous demande de nous

23 R. Rorty, Contingence, Irony, Solidarity, Cambridge, Cambridge University Press, 1989, p. 133, trad. M. K.

24 « Fidèle et infidèle, comme vous avez raison! Je me vois souvent passer très vite devant le miroir de la vie, comme la silhouette d'un fou (à la fois comique et tragique) qui se tue à être infidèle par esprit de fidélité " (J. Derrida, De quoi demain... Dialogue, op. cit., p. 14-15).

${ }^{25}$ Cf. J. Derrida, L'Université sans condition, Paris, Galilée, 2001, p. 26-36.

26 Cette élision se référant également à ce qu'il voulait transmettre dans un message crypté en se disséminant dans l'écriture. Ainsi, dans une courte préface précédant Envois, il avertit le lecteur qu'il a effacé certains fragments de son texte et marqué ces lieux d'effacement par un blanc de 52 signes. "Il peut s'agir d'un nom propre ou d'un signe de ponctuation, de l'apostrophe seulement qui remplace une lettre é l i d é e [...] " (J. Derrida, La carte postale. De Socrate à Freud et au-delà, Paris, Flammarion, 1980, p. 8. C'est moi qui souligne). Dans ce langage hanté par le secret, une lettre élidée peut référer à l'un des noms propres de Jackie Derrida, Élie, qui lui est donné au moment de la 
limiter à l'essentiel : de le suivre au prix de l'imiter. De l'autre côté, je fais, en l'imitant, comme si j'avais à prononcer ce que j'écris ici même à la mémoire de Derrida, en me souvenant de sa parole que je crois e n t e ndre de façon a u t o n o me. Commenter Derrida, écrire a prè s lui revient à $p$ a rle $r$ de $r$ rida, parler son français. L'écriture derridienne a besoin d'un coup de théâtre et d'une certaine performance. Elle passe par le pouvoir de la parole : sa magie performative et sa performativité magique. Que cette parole ne puisse se dire que dans l'écriture, tout commentaire, si elle se veut fidèle, ne peut que la réciter en la rejouant selon sa propre mise en scène.

Tout se passe comme si le style de Derrida, la manière dont il veut s'écrire, allait à l'encontre de ce qu'il d i s a it lors de sa conférence "Signature, événement, contexte ", où il se prenait à déconstruire la $\mathrm{s} p \mathrm{e} e \mathrm{ch}$ - a c t th e ory d'Austin ${ }^{27}$; comme si l'écriture derridienne ne se lisait pas, mais restait entièrement à $\mathrm{s}$ e dire et que cette performativité, cette mise en scène techno-phonétique et technopoétique dût contresigner ce que Derrida définissait comme dissémination. Or l'entente passe par ce qui reste plus à sentir qu'à voir. II suffit de lire les derniers séminaires de Derrida où l'émotion demande manifestement à être entendue 28 .

circoncision sans être inscrit à l'état civil. Il l'évoque pour la première fois dans Circonfession au moment où il fait allusion à son frère, Paul Moïse, qui est mort un an avant sa naissance, " ce qui dut faire de moi pour elle [sa mère], pour eux, un précieux mais si vulnérable intrus, un mortel de trop, Élie aimé à la place d'un autre " (J. Derrida, Circonfession, op. cit., p. 52-53). D'autre part, l'élision d'Élie fait penser au projet d'un livre que Derrida n'aurait jamais écrit. L'un des carnets qu'il entame en 1976, au moment où les traces autobiographiques de son écriture deviennent plus visibles quoique chiffrées, est intitulé " Le livre d'Élie " (B. Peeters, Derrida, Paris, Flammarion, 2010, p. 359).

${ }^{27}$ Cf. J. Derrida, Marges de la philosophie, Paris, Seuil, 1972, p. 365-393

28 J. Derrida, L'animal que donc je suis, Paris, Galilée, 2006 ; Idem, Séminaire La bête et le souverain. Volume I (2001-2002), Paris, Galilée, 2008 ; Idem, La bête et le souverain. Volume II (2002-2003), Paris, Galilée, 2010. 
«Surtout pas de récit »

Mais ce flair théâtral, ce coup de théâtre sur la scène philosophique, permet également de jouer l'autobiographique tout en déjouant " la loi du genre ». Tout en restant fidèle à Derrida et à ses métaphores, il s'agit de suivre les traces de l'autobiographique et de porter l'attention sur la dés-espérance avec laquelle il tenait à se sauver par son lexique. En gros, il serait donc question de retrouver l'homme Derrida, cet " animal autobiographique " qui se suit lui-même dans cette aventure textuelle que les premiers lecteurs américains de Derrida, beaucoup plus que Derrida lui-même et avant lui, ont reconnue sous le nom de déconstruction.

Or, en évoquant ce coup de théâtre sur la scène philosophique, j'aimerais également donner un coup d'envoi. Ma lecture de Derrida s'inscrit dans un projet de recherche plus large qui porte sur ce que j'appelle "philosophie auto/biographique " et la relation entre la pensée et la vie. En gros, il s'agit de lire des textes philosophiques, théoriques ou ceux dont le statut balance entre philosophie et littérature, comme si ces textes étaient une manière d'écrire le soi ou de vivre sa vie. Cela non pas pour en faire une lecture naïve et quelque peu stérile qui aurait à prouver qu'une expérience, un événement ou un fait avait précédé tel ou tel concept. Il s'agit plutôt, dans la veine derridienne, de suivre une trace de vie, mais aussi une vie comme trace : apprendre à reconnaître des vies dans le mouvement général de la pensée afin de la saisir à ras de terre même. Autrement dit, afin de la profaner : la rendre à l'usage de tous et montrer ce que la pensée, au demeurant, a de matérie ${ }^{29}$.

\footnotetext{
${ }^{29}$ En ce sens, je reste un fidèle lecteur de Bataille et de son matérialisme, tout en considérant la manière dont il met sa vie dans ses écrits comme exemplaire. Cf. M. Krzykawski, "Après coup. De l'effet-Bataille à l'aveu de Bataille ", [dans :] Cahiers Bataille, 2016, n 3, p. 77-93 ; Idem, "Les oiseaux de Bataille », [dans :] Romanica Silesiana, 2016, n 11, p. 242-253 ;
} 
Dans une perspective plus large, il serait question de retrouver le sens de la philologie après le poststructuralisme qui a un peu bêtement rejeté la question autobiographique, tout en reconnaissant, après coup, ce que le terme de poststructuralisme a d'équivoque et compte tenu des différences structurelles, institutionnelles, culturelles, historiques et sociales entre les champs intellectuels français et anglo-américain qui ont toutes participé à la production du savoir au sein de ce qu'on définit volontiers aujourd'hui comme " humanités ", et qui traversent désormais la scène théorique. Être philologue avec une conscience aiguë du moment poststructuraliste dans l'histoire de la pensée contemporaine française, qui est aussi l'histoire de sa réception hors la France $^{30}$, est une double réponse. Il faut répondre "à ", mais aussi répondre " de " et " pour " la manière dont il nous revient à hériter du poststructuralisme. Si c'est encore Derrida qui me souffle le langage permettant de répondre, c'est-à-dire de " filtrer, cribler, critiquer, [...] trier $\|^{31}$, conformément aux structures d'héritage qu'il évoque dans Spectres de Marx, c'est Bernard Stiegler qui $\mathrm{m}^{\prime}$ incite à relire le poststructuralisme comme ensemble de $\mathrm{ph}$ a rmaka dans leur rapport à la bêtise et au savoir ${ }^{32}$. Cela revient, en l'occurrence, à récuser la bêtise poststructuraliste et, en même temps, à prendre position depuis le poststructuralisme, ce "depuis " nous permettant d'un côté de mieux saisir l'après-poststructuralisme

Idem, "Le petit secret de Georges Bataille », [dans :] Alkemie, 2015, $\mathrm{n}^{\circ}$ 15, p. 153-168; Idem, " The Amorous Frenzy of Things. Georges Bataille's Basely Material Love ", [dans :] M. Gratzke, A. Malinowska (dir.), The Materiality of Love, New York-London, Routledge, 2017. À paraître.

${ }^{30}$ Cf. F. Cusset, French Theory, Foucault, Derrida, Deleuze et Cie et les mutations de la vie intellectuelle aux États-Unis, Paris, La Découverte, 2003 ; S. Lotringer, S. Cohen (dir.), French Theory in America, New York, London, Routledge, 2001.

31 J. Derrida, Spectres de Marx, Paris, Galilée, 1993, p. 40.

32 Cf. B. Stiegler, États de choc. Bêtise et savoir au XXIe siècle, Paris, Mille et une nuits, 2012. 
tel qu'il nous vie n t ici et maintenant et, de l'autre côté, de mieux $r$ e ve $n$ i $r$ en arrière, à l'époque d'avant le poststructuralisme pour mieux voir ce qui est devant nous.

Dans une perspective plus restreinte, trois auteurs m'intéressent dans ce projet, trois auteurs que tout diffère par leurs tempéraments et par la manière dont ils positionnent l'autobiographique dans leurs écrits. Ce sont Georges Bataille, Maurice Blanchot et Jacques Derrida : l'exhibition presque totale, l'effacement jusqu'à l'autointerdiction et l'incapacité de se dire à travers une anamnèse. Trois auteurs, deux paires d'amis : Bataille, Blanchot et Blanchot, Derrida. La question philologique, on le voit, est également la question amicale. En fait, il s'agit de retrouver le nouveau sens de ce que les Grecs définissaient comme philia.

C'est dans l'autobiographique qui entre, avec ces trois auteurs, sur la scène philosophique que l'on peut saisir le " flou sentimental et émotionnel » qui est au cœur même des concepts philosophiques. Or l'aborder depuis la philosophie et non pas depuis la littérature rencontre le problème de légitimité. Qu'il me soit permis de le dire vite : si le droit à l'autobiographie passe nécessairement par la littérature, c'est d'un côté parce qu'il nous est toujours difficile de penser l'autobiographique hors du récit. Il suffit de rappeler Lejeune qui a défini l'autobiographie comme un "récit rétrospectif en prose qu'une personne réelle fait de sa propre existence, lorsqu'elle met l'accent sur sa vie individuelle, en particulier sur l'histoire de sa personnalité ${ }^{33}$. De l'autre côté, nous les autres postmodernes restons plutôt postromantiques dans notre manière de penser la pensée. En romantisant la pensée philosophique, nous prenons pour une évidence même que la vie de celui qui pense, dans sa contingence, est extérieure à la pensée. La question est donc de savoir

$\overline{33} \mathrm{Ph}$. Lejeune, Le pacte autobiographique, Paris, Seuil, 1975, p. 14. 
si le philosophe, malgré l'effort entrepris par Nietzsche dans Ecce Homo, a droit à la vie. L'enjeu principal de la philosophie auto/biographique est donc de retrouver la place de l'autobiographique dans le philosophique et de dissocier l'autobiographique du récitatif. Jean Luc Nancy remarque que "le récit met en œuvre et en jeu son récitant : pas de récit sans récitation et pas de récitation sans récitant. Ce dernier ne se présente jamais que comme distinct du récit, lors même qu'il en est l'objet, comme il est censé l'être dans une autobiographie $»^{34}$. Or, puisque la philosophie ne prend jamais la forme du récit, il faudrait, pour saisir la philosophie auto/biographique, suivre une nouvelle forme d'énonciation de l'autobiographique. Pas de pacte autobiographique dans la philosophie, sinon celui de ne rien dire sur soi. Inutile donc de se servir de la boîte d'outils élaborés dans le champ littéraire, même si l'autobiographique dans le philosophique se rapproche, par la force des choses, du littéraire. Poser la question autobiographique ainsi, la poser autrement que par l'intermédiaire du récit pour voir quelle pourrait être la place sur la scène philosophique de ce que Roland Barthes, quatre ans avant de publier son autobiographie, a défini comme " biographèmes ${ }^{35}$, revient peut-être à aller plus loin que Nancy, même si cette question doit être posée dans le sillage de sa méditation sur la manière dont la philosophie et la littérature se demandent la vérité l'une à l'autre ${ }^{36}$.

\footnotetext{
34 J.-L. Nancy, Demande, Paris, Galilée, 2015, p. 59.

35 « Si j'étais écrivain, et mort, comme j'aimerais que ma vie se réduisît, par les soins d'un biographe amical et désinvolte, à quelques détails, à quelques goûts, à quelques inflexions : des "biographèmes", dont la distinction et la mobilité pourraient voyager hors de tout destin et venir toucher, à la façon des atomes épicuriens, quelque corps futur, promis à la même dispersion "(R. Barthes, Sade, Fourier, Loyola, Paris, Seuil, 1971, p. 13).

${ }^{36}$ J.-L. Nancy, Demande, op. cit., p. 9-10.
} 


\section{Fil conducteur, fil pénien}

Vu ces problèmes de méthode, Circonfession est une entreprise d'écriture exemplaire. L'enjeu de ce texte, publié en 1990, se traduit le mieux par les événements qui participent à sa naissance. Au départ, Circonfession devait être un texte d'accompagnement inclus dans le livre sur Derrida, écrit par Geoffrey Bennington qui a proposé de créer " une sorte de "logiciel Derrida" et de faire une analyse de son œuvre sans aucune citation de lui ${ }^{37}$. Ayant reçu le manuscrit de "Derridabase ", car tel était le titre du texte benningtonien, Derrida se voit en quelque sorte circonscrit. II devient un Derrida théorique, un Derrida mis en boîte, pas loin de la boîte d'outils par laquelle Gilles Deleuze a défini la théorie en $1972^{38}$. La tentative de réduire sa pensée à la pure fonctionnalité d'un logiciel lui inspire de l'inquiétude. II ne lui reste qu'à " déconstruire de l'intérieur l'ouvrage qui lui est consacré ${ }^{39}$. Derrida décide de jouer la carte autobiographique et contresigner le texte su r lui par un autre qui échappe au logiciel de Bennington. Techniquement parlant, il écrit un texte qui se compose de 59 paragraphes, chacun étant une phrase aussi longue que le permettait le programme MacWrite qu'il utilisait, tandis que le nombre des paragraphes répond à l'âge qu'il avait au moment où il a commencé à écrire Circonfession en 1989. Dans le livre, le texte de Bennington est placé au-dessus de celui de Derrida, lequel occupe un tiers de chaque page. Le texte su r lui l'est également dans le sens spatial, ce qu'il ne manque pas de souligner.

\footnotetext{
${ }^{37}$ Cité par B. Peeters, Derrida, op. cit., p. 495.

38 « C'est ça, une théorie, c'est exactement comme une boîte à outils. [...] II faut que ça serve, que ça fonctionne. Et pas pour soi-même " ("Les intellectuels et le pouvoir ", entretien avec M. Foucault, [dans :] M. Foucault, Dits et écrits I. 1954-1975, Paris, Gallimard, 2001, p. 1177).

${ }^{39}$ B. Peeters, Derrida, op. cit., p. 495.
} 
et il a décidé, par cette circoncision rigoureuse, de se passer de mon corps, du corps de mes écrits pour produire en somme la "logique » ou la "grammaire ", la loi de production de tout énoncé passé, présent et pourquoi pas futur que je pourrai avoir signé [...] si bien que me voilà privé d'avenir, plus d'événements à venir de moi [...] sauf si j'écris ici même, sauve qui peut, cessant d'être sous sa loi, des choses improbables qui déstabilisent, déconcertent, surprennent à leur tour le programme de G., des choses qu'en somme il n'aura pas pu [...] reconnaître, nommer, prévoir, produire, prédire, unpredictable things pour lui survivre. ${ }^{40}$

Tout se passe comme si Derrida se sentait contraint de se sauver contre sa propre pensée qui s'écrit sur lui dès que celle-ci est mise à l'usage des autres, d'ailleurs conformément au principe de l'itérabilitié qui, selon Derrida luimême, seul permet de garder la propriété (et la propreté) du nom propre. Le propre doit se perdre dans la répétition. Or la contresignature du texte sur lui, servant à la réappropriation de sa propre pensée, passe par le corps qui est aussi son corpus qu'il ne veut pourtant pas rompre et donner à $G$. comme Jésus à ses disciples. Le corps/le corpus derridien est en même temps le terrain et l'objet de la lutte. II en est le s e n s:

ceci est mon corpus, l'ensemble des phrases que j'ai signées dont il n'a littéralement pas cité une, pas une dans sa littéralité, ce fut le choix, il s'en explique, un choix étrange quand on écrit un livre s u r quelqu'un qui écrit des livres, or il n'a pas gardé un seul fragment intact de mon corpus et s'il en a sectionné ou prélevé quelques morceaux, c'est juste pour ne pas les garder, pour les laisser comme des peaux inutiles à l'intelligence de mes textes. ${ }^{41}$

Ce duel textuel entre l'auteur et son commentateur, dont il approuve et autorise le commentaire, est une étrange lutte pour la paternité ou l'auctorialité de la pensée, même s'il ne peut s'agir que de sauver des traces de soi dans son propre texte. Rien d'étrange que Derrida se défende avec une arme on ne peut plus phallique et n'hésite pas à engager dans cette lutte corporelle son propre pénis. C'est la « condition d'un pacte ineffaçable

40 J. Derrida, Circonfession, op. cit., p. 31-32.

41 Ibidem, p. 28-29. 
pour le seul philosophe à ma connaissance qui, accueilli plus ou moins - dans l'institution académique [...] aura osé décrire son pénis, comme promis, de façon concise et détaillée ${ }^{42}$. La déconstruction du texte sur soi, en l'occurrence, passe par une exhibition totale. En s'exhibant, Derrida exhibe son écriture comme on exhibe une pièce d'identité devant l'autorité. Il s'exhibe devant lui-même.

L'exhibition, dans cette double action de se produire et de se montrer, est ce qui déclenche l'autobiographique. Dans Circonfession, dès la première phrase, il s'agit de voir ce que "parler cru veut dire ", de retrouver "le vocable cru » et "lui disputer ainsi le cru » ${ }^{43}$. D'ailleurs, ce goût de l'exhibition, de parler cru, a pu se voir déjà dans "Envois", première partie de La carte postale, où l'interprétation de l'illustration de Matthew Paris, représentant Socrate écrivant devant Platon, implique Derrida et celle avec qui il vit une histoire d'amour passionnée :

je vois Plato bander dans le dos de Socrates et l'ubris insensée de sa queue, une érection interminable, disproportionnée, traverser comme une seule idée la tête de Paris et la chaise du copiste avant de glisser doucement, toute chaude encore, sous la jambe de Socrates, en harmonie ou symphonie de mouvement avec ce faisceau de phallus, les pointes, plumes, doigts, ongles et grattoirs, les écritoires qui s'adressent dans la même direction. La di-rection, le diérection de ce couple, de ces vieux fous, de ces galopins à cheval, c'est nous. ${ }^{44}$

C'est Derrida lui-même qui confie au traducteur américain de son texte comment il s'y dissémine. Dans " j'accepte» il faut lire "Jacques " tandis que " sept " répond au nombre des lettres du nom Derrida ${ }^{45}$. Si l'on veut suivre cet indice, on pourrait croire que So crates, et non pas Socrate, répondrait à Sylviane ( 8 lettres), tandis que

\footnotetext{
42 Ibidem, p. 110.

43 Ibidem, p. 7.

44 J. Derrida, La carte postale, op. cit., p. 22-23.

45 Cf. A. Bass, "Translator's Introduction. L Before K », [dans :] J. Derrida, The Post Card. From Socrates to Freud and Beyond, A. Bass (trad.), Chicago-London, The University of Chicago Press, 1987.
} 
P I a t o, prononcé à la française, pourrait correspondre au plateau où tout se joue : la scène de l'écriture se double avec la scène d'amour loin d'être platonique (phase plateau ?). Or, cette exhibition, qui frôle l'exhibitionnisme, fût-il crypté, peut aussi inspirer du malaise, dès qu'on s'exhibe devant une bête. II en est ainsi dans la fameuse scène dépeinte dans L'animal que donc je suis, où Derrida se décrit nu devant sa chatte qui l'observe:

J'ai du mal à réprimer un mouvement de pudeur. Du mal à faire taire en moi une protestation contre l'indécence. Contre la malséance qu'il peut y avoir à se trouver nu, le sexe exposé, à poil, devant un chat qui vous regarde sans bouger, juste pour voir. ${ }^{46}$

" "Sa" chatte ", cette " petite chatte noire ", qu'il rapporte à celle retrouvée chez Montaigne ou celle dans Alice au pays des merveilles, lui inspire de la honte. D'un côté, ce rapprochement entre le vécu et le lu débouche sur un axiome philosophique fort connu : " L'animal nous regarde, et nous sommes nus devant lui. Et penser commence peut-être là ${ }^{47}$. Or, de l'autre côté, cette honte n'empêche pas Derrida, tout impudiquement, de jouer avec cette chatte comme on joue avec les mots. C'est un autre gage de théâtralité qui fait parler l'autobiographique.

Que ce goût de l'autobiographique engage Derrida jusqu'à son sexe, pour ainsi dire, Circonfession en est l'exemple le plus frappant, car tout s'y joue autour de sa circoncision qu'il reconnaît après-coup afin même de relancer, comme on relance lors d'un jeu, la confession telle qu'il l'a cryptée jusque-là dans s a langue à lui. Cette reconnaissance ne va pourtant pas sans problèmes. Le souvenir de la circoncision doit permettre de fuir la circonscription qui se produit sur lui, dans le texte de Bennington. Le Derrida circoncis l'emporte sur le Derrida

$\overline{46}$ J. Derrida, L'animal que donc je suis, op. cit., p. 18.

47 Ibidem, p. 50. 
circonscrit. Or, quoique la circoncision soit le symbole majeur de l'appartenance à une communauté particulière, il serait trop facile de voir en Derrida un penseur juif ou un crypto-juif pratiquant secrètement sa religion à l'instar d'un marrane, ce juif espagnol converti au catholicisme, et de mettre en avant sa judéité présumée qui nous permettrait de déchiffrer le secret. C'est que le souvenir de la circoncision est le souvenir de ce dont on ne peut porter aucun souvenir. La circoncision, bien que réellement subie, ne peut donc pas faire l'objet d'une confession, et par conséquent d'une autobiographie sous la forme d'un récit. Et c'est cette impossibilité même que Derrida veut confesser en en faisant le gage de son duel avec celui qui veut le circonscrire. II est important de souligner qu'en fin de compte Derrida fait sa circonfession non pas pour instituer le père (se déclarer juif), mais pour garder le souvenir de sa mère. Lutter pour la paternité de son texte, fût-ce avec son pénis, ne revient pas à se reconnaître dans la figure du père. Circonfession doit se lire surtout comme l'aveu d'un amour filial du fils pour sa mère, mourante au moment où il écrit.

moi qui, entre autres remords à l'endroit de ma mère, me sens bien coupable de publier sa fin, d'en exhiber les derniers souffles et pis encore, à des fins que d'aucuns pourraient juger littéraires, au risque d'ajouter un exercice douteux à la série "l'écrivain et sa mère ", soussérie "la mort de la mère ", et que faire, ne me sentirais-je pas aussi coupable, ne le serais-je pas en vérité si j'écrivais ici de moi sans garder la moindre trace d'elle. ${ }^{48}$

La judéité derridienne, s'il y en a, passe nécessairement par le corps-à-corps avec la mère. Le fils n'accepte d'avouer sa judéité que pour la parjurer. II fait comme s'il fallait réinventer sa propre circoncision et écrire, non pas avec son stylo/pénis, mais avec son prépuce qu'il prend pour un anneau liant le fils à sa mère ; comme si, faute de pouvoir arriver au moment d'avant la coupure, on ne

48 J. Derrida, Circonfession, op. cit., p. 38. 
pouvait écrire qu'à partir du reste : se lover dans ce hors le langage, dire ce reste, dire ce qui reste à dire dans " la vieille neuve langue ${ }^{49}$, ce qui revient à disséminer le reste dans la langue commune, y laisser des traces de sa propre semence. Or, ce moment d'avant la coupure, dont je ne peux pas me souvenir, qui ne m'appartient donc pas, est celui d'avant la mémoire. Ce n'est même pas quelque chose qui s'oublie, comme la foi qu'un marrane pratique secrètement jusqu'à l'oublier. C'est plutôt " l'oubli de l'oubli, de l'oubli dont il ne reste rien ${ }^{50}$ que Derrida évoque dans une émission de France Culture de 1986. La sur-mémoire qui bloque le récit, sans pourtant éliminer l'inscription autobiographique, œuvre également pour ce qui reste hors la mémoire.

Si immense que soit l'héritage de Derrida, il nous a également légué, en l'ouvrant, ce lieu depuis lequel le lien entre l'autobiographique et le philosophique apparaît comme une évidence même qui reste pourtant entièrement à être entendue. Derrida nous a rappelé que faire de la philosophie n'est possible qu'en s'écrivant dans une éternelle remémoration qui est également une reconstitution de soi. Certes, il l'a fait d'une manière singulière qui n'appartient qu'à lui. II s'est disséminé dans ses propres concepts et il y reste à re m e $\mathrm{m}$ b re r. J'ose dire que, depuis Derrida, il faudrait peut-être parler de I'autobiosophie et la philographie pour souligner que le désir $d^{\prime}$ " apprendre à vivre enfin " $^{51}$ et celui d'écrire sont inséparables et déterminent le s e $\mathrm{n} s$ de la philosophie. Le fil pénien qui a orienté ma lecture de Circonfession, et qui est, en l'occurrence, la manière d'engager dans l'écriture son propre pénis, apparaît comme

49 J. Derrida, "Desceller ("la vieille neuve langue") ", [dans :] Idem, Points de suspension, op. cit., p. 123-140.

$50 \mathrm{~J}$. Derrida, "II n'y a pas le narcissisme », [dans :] Idem, Points de suspension, op. cit., p. 221.

$51 \mathrm{~J}$. Derrida, Apprendre à vivre enfin. Entretien avec Jean Birnbaum, Paris, Galilée, 2005. Cf. J. Derrida, Spectres de Marx, op. cit., p. 13-18. 
une des multiples manières derridiennes de laisser (et se suivre après) ses traces. Il fait partie du tuf autobiographique de l'écriture de Derrida ou, pour le dire comme lui, constitue le " théâtre intérieur $~^{52}$ de ses concepts philosophiques.

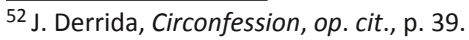




\section{bibliliographie}

Barthes R., Sade, Fourier, Loyola, Paris, Seuil, 1971.

Bass A., "Translator's Introduction. L Before K ", [dans :] J. Derrida, The Post Card. From Socrates to Freud and Beyond, A. Bass (trad.), ChicagoLondon, The University of Chicago Press, 1987.

Cusset F., French Theory, Foucault, Derrida, Deleuze et Cie et les mutations de la vie intellectuelle aux États-Unis, Paris, La Découverte, 2003.

Deleuze G., "Les intellectuels et le pouvoir », entretien avec Michel Foucault, [dans :] M. Foucault, Dits et écrits I. 1954-1975, Paris, Gallimard, 2001.

Derrida J., L'animal que donc je suis, Paris, Galilée, 2006.

Derrida J., Apprendre à vivre enfin. Entretien avec Jean Birnbaum, Paris, Galilée, 2005.

Derrida J., Séminaire La bête et le souverain. Volume I (2001-2002), Paris, Galilée, 2008.

Derrida J., La bête et le souverain. Volume II (2002-2003), Paris, Galilée, 2010.

Derrida J., La carte postale. De Socrate à Freud et au-delà, Paris, Flammarion, 1980.

Derrida J., Circonfession, [dans :] G. Bennington, Jacques Derrida, Paris, Seuil, 1991.

Derrida J., Roudinesco É., De quoi demain... Dialogue, Paris, Flammarion, 2001.

Derrida J., " Desceller ("la vieille neuve langue") ", [dans :] Idem, Points de suspension, Paris, Galilée, 1992.

Derrida J., "La différance ", [dans :] Idem, Marges de la philosophie, Paris, Minuit, 1972.

Derrida J., "Freud et la scène d'écriture ", [dans :] Idem, L'écriture et la différence, Paris, Seuil, 1967.

Derrida J., Feu la cendre, Paris, Des Femmes, 1986.

Derrida J., " "Il faut bien manger" ou le calcul du sujet ", entretien avec Jean-Luc Nancy, [dans :] Idem, Points de suspension, Paris, Galilée, 1992.

Derrida J., "II n'y a pas le narcissisme ", [dans :] Idem, Points de suspension, Paris, Galilée, 1992.

Derrida J., Marges de la philosophie, Paris, Seuil, 1972.

Derrida J., Mémoires pour Paul de Man, Paris, Galilée, 1988.

Derrida J., "La pharmacie de Platon », [dans :] Idem, La Dissémination, Paris, Seuil, 1972.

Derrida J., Signéponge, Paris, Seuil, 1988.

Derrida J., Spectres de Marx, Paris, Galilée, 1993.

Derrida J., Sur parole. Instantanés philosophiques, Paris, Éditions de l'Aube, 1999.

Derrida J., L'Université sans condition, Paris, Galilée, 2001.

Peeters B., Derrida, Paris, Flammarion, 2010.

Krzykawski M., "The Amorous Frenzy of Things. On Bataille's Bawdy Lyricism ", [dans :] M. Gratzke, A. Malinowska (dir.), The Materiality of Love, New York, London, Routledge, 2017. À paraître. 
Krzykawski M., "Après coup. De l'effet-Bataille à l'aveu de Bataille ", [dans :] Cahiers Bataille, 2016, $\mathrm{n}^{\circ} 3$.

Krzykawski M., "La mélancolie de la déconstruction. Ce qui reste de Jacques Derrida », [dans :] Alkemie, 2017, no 19.

Krzykawski M., "Les oiseaux de Bataille », [dans :] Romanica Silesiana, 2016, no 11.

Krzykawski M., "Le petit secret de Georges Bataille ", [dans :] Alkemie, 2015, n० 15.

Lejeune Ph., Le pacte autobiographique, Paris, Seuil, 1975.

Lotringer S., Cohen S. (dir.), French Theory in America, New York, London, Routledge, 2001.

Nancy J.-L., Demande, Paris, Galilée, 2015.

Rorty R., Contingence, Irony, Solidarity, Cambridge, Cambridge University Press, 1989.

Stiegler B., États de choc. Bêtise et savoir au XXIe siècle, Paris, Mille et une nuits, 2012.

\section{abstract}

\section{Derrida at Work. On Circumfession and Beyond}

The aim of this article is to discuss the autobiographical inscription that dwells in Jacques Derrida's writing. Derrida's " overmemory ", that blocks autobiography as a récit, seems to be the trigger for the singular and somehow emotional style of his writing through which he saves himself inside his own philosophy based, among others, on the principle of dissemination. Circumfession, that I read through what I call " penile thread " [fil pénien], shows different strategies to preserve some traces of Derrida's p r o p e r name.

\section{keywords}

auto/biography, memory, Jewishness, masculinity

\section{michał krzykawski}

Maître de conférences à l'Institut des langues romanes et de la traduction de I'Université de Silésie. II a publié L'Effet-Bataille. De la littérature d'excès à l'écriture. Un texte-lecture (2011) et une trentaine d'articles consacrés à Bataille, Blanchot, Derrida, Nancy, Bailly et Agamben. II travaille actuellement sur la communauté et l'amitié dans la pensée contemporaine française (projet de recherche financé par le Centre National de la Science en Pologne), ainsi que sur la condition animale. Son dernier livre (en polonais), L'autre et le commun. Trente-cinq ans de philosophie française, vient de paraître. 